\title{
Cytokine Autoantibodies Are Associated with Infection Risk and Self-Perceived Health: Results from the Danish Blood Donor Study
}

\author{
Jakob H. von Stemann ${ }^{1}$ (D) - Ole B. Pedersen ${ }^{2} \cdot$ Henrik Hjalgrim $^{3,4} \cdot$ Christian Erikstrup $^{5} \cdot$ Henrik Ullum $^{1}$. \\ Lise W. Thørner ${ }^{1} \cdot$ Margit AH. Larsen ${ }^{1} \cdot$ Kristoffer S. Burgdorf $^{1} \cdot$ Erik Sørensen $^{1} \cdot$ Morten B. Hansen ${ }^{1}$. \\ Sisse R. Ostrowski ${ }^{1}$
}

Received: 12 September 2019 / Accepted: 6 January 2020 / Published online: 15 January 2020

(C) The Author(s) 2020

\begin{abstract}
The presence of naturally occurring cytokine-specific autoantibodies (c-aAb) in humans is well established, as well as associations to selected pathologies. However, the overall influence of $\mathrm{c}-\mathrm{aAb}$ on immunocompetence remains largely unknown. In this paper, we performed a large-scale investigation of $\mathrm{c}-\mathrm{aAb}$ association with infection risk. A cohort of healthy Danish blood donors was screened for $\mathrm{c}-\mathrm{aAb}$ against IL-1 $\alpha$, IL-6, IL-10, IFN $\alpha$, and GM-CSF using a Luminex-based multiplex assay, and results were linked to data from the Danish National Prescription Registry. The filing of an antimicrobial prescription following c-aAb measurement was used as a proxy for impaired immunocompetence. We found that c-aAb against pro-inflammatory cytokines IFN $\alpha$ and GM-CSF tended to associate with increased risk of prescription filings in women, whereas antibodies against antiinflammatory IL-10 were associated with a lower predicted risk of antimicrobial prescriptions, as well as higher self-perceived health scores. We also observed an association of cumulative c-aAb presence with prescription risk. Our data show that cytokine autoantibodies in healthy individuals associate with various proxies for immunomodulation, with the exact association dependent on the pattern of pro- or anti-inflammatory cytokines targeted. This suggests that c-aAb may express cytokine-modulatory properties in healthy individuals and may be critical to further investigate as biomarkers of immunodeficiency.
\end{abstract}

Keywords Autoimmunity $\cdot$ cytokine autoantibodies $\cdot$ IL-10 $\cdot$ IFN $\alpha \cdot$ blood donors $\cdot$ epidemiology

Electronic supplementary material The online version of this article (https://doi.org/10.1007/s10875-020-00744-3) contains supplementary material, which is available to authorized users.

Jakob H. von Stemann

jakob.hjorth.von.stemann@ @regionh.dk

1 Department of Clinical Immunology, Rigshospitalet, Copenhagen University Hospital, Blegdamsvej 9, 2100 Copenhagen, Denmark

2 Department of Clinical Immunology, Næstved Sygehus, Næstved, Denmark

3 Epidemiology Research, Statens Serum Institut, Copenhagen, Denmark

4 Department of Hematology, Rigshospitalet, Copenhagen University Hospital, Copenhagen, Denmark

5 Department of Clinical Immunology, Aarhus University Hospital, Aarhus, Denmark

$\begin{array}{ll}\text { Abbreviations } \\ \text { BMI } & \text { Body mass index } \\ \text { C-aAb } & \text { Cytokine-specific autoantibodies } \\ \text { CI } & \text { Confidence interval } \\ \text { DBDS } & \text { The Danish Blood Donor Study } \\ \text { DNPR } & \text { Danish National Prescription Registry } \\ \text { GM-CSF } & \text { Granulocyte-macrophage } \\ & \text { colony-stimulating factor } \\ \text { HR } & \text { Hazard ratio } \\ \text { IL } & \text { Interleukin } \\ \text { IFN } & \text { Interferon } \\ \text { MCS } & \text { Mental component score } \\ \text { MFI } & \text { Median fluorescence intensity } \\ \text { OC } & \text { Oral contraceptives } \\ \text { OR } & \text { Odds ratio } \\ \text { PCS } & \text { Physical component score } \\ \text { SD } & \text { Standard deviation }\end{array}$




\section{Introduction}

Cytokines are pivotal signaling molecules in the immune system balancing pro- and anti-inflammatory immune responses [1]. There is emerging evidence that naturally occurring cytokine-specific autoantibodies (c-aAb) may critically influence immune competence [2-5].

The influence of $\mathrm{c}-\mathrm{aAb}$ on the systemic cytokine networks remains mostly unknown, though multiple studies have linked specific c-aAb to various pathologies and opportunistic infections [6-8]. Most notably, granulocytemacrophage colony-stimulating factor (GM-CSF) $\mathrm{c}-\mathrm{aAb}$ have been identified as a causal factor for pulmonary alveolar proteinosis (PAP), a rare and debilitating respiratory disease $[9,10]$. A range of $\mathrm{c}-\mathrm{aAb}$ have also been labeled as phenotypic classifiers for various primary immunodeficiencies (including GM-CSF c-aAb for PAP) by the international union of immunological societies [11]. Conversely, c-aAb against pro-inflammatory interleukins (IL) have been associated with improved prognosis or reduced symptoms in some autoimmune diseases, such as rheumatoid arthritis and Sjögrens syndrome indicating that ultimately the biologic effect of c-aAb depends on the specific pathological context combined with the $\mathrm{c}-\mathrm{aAb}$ specificity $[12,13]$.

The trigger for $\mathrm{c}-\mathrm{aAb}$ production is debated. Some studies indicate that the genetic framework may promote $\mathrm{c}-\mathrm{aAb}$ (e.g., specific variants of the antigen-presenting human leukocyte antigen or mutations of the autoimmune regulator gene, AIRE), in addition to microbe-induced loss of tolerance, through mechanisms such as molecular mimicry $[14,15]$.

We have previously reported that detectable levels of $\mathrm{c}-\mathrm{aAb}$ are common among healthy individuals, with high levels being associated with older age and male sex. Furthermore, in healthy individuals, high levels of IL-6 c-aAb are associated with low C-reactive protein (CRP) levels, indicating a possible functional effect of naturally occurring, high-titer $\mathrm{c}-\mathrm{aAb}$ [16].

The aim of the present study was to investigate the association between available indicators of $\mathrm{c}-\mathrm{aAb}$ mediated immune modulation, evidenced by infectious risk and self-perceived health. We hypothesized that high-titer levels of $\mathrm{c}-\mathrm{aAb}$ may inflict immunomodulation to a degree that would either increase or reduce infectious risk and self-perceived health. Thus, we linked our previously $\mathrm{c}-\mathrm{aAb}$ screened cohort of healthy blood donors to health register and questionnaire data to assess possible outcomes of $\mathrm{c}-\mathrm{aAb}$-mediated immunomodulation, with antimicrobial prescription filings and self-perceived health as endpoints. To our knowledge, this is the largest study of the biologic and potentially immunomodulatory effects of $\mathrm{c}-\mathrm{aAb}$ in a healthy population to date.

\section{Materials and Methods}

\section{Study Population}

The study includes 8972 blood donors enrolled in 2010 in the first iteration of The Danish Blood Donor Study (DBDS), a nationwide prospective cohort study on blood donor health $[17,18]$. All participants were healthy at the time of inclusion and between 18 and 67 years of age. At the day of inclusion, participants completed a written health questionnaire, and a plasma sample was retained for biomarker analysis, which included $\mathrm{c}-\mathrm{aAb}$ measurements. Participants were censored from analysis in this study in the event of missing data for any dependent or independent variable.

\section{Coding of Dependent and Independent Variables}

\section{Epidemiological Covariates}

Based on the questionnaire, information on potential covariates including participant body mass index (BMI), smoking status, and usage of combined oral contraceptives (OC) was obtained. BMI was calculated as self-reported weight $(\mathrm{kg}) /$ self-reported height $(\mathrm{m})$ squared and was censored in the event of unrealistic anthropometric outliers (heights $<1,5 \mathrm{~m}$ and $>2,1 \mathrm{~m}$, weight $<45 \mathrm{~kg}$ and $>160 \mathrm{~kg}$ ). Participants were coded as smokers if they identified as currently smoking any number of cigarettes at the time of inclusion. Age was defined at sample date. Usage of $\mathrm{OC}$ was defined at the time of inclusion.

Charlson comorbidity score [19] was calculated based on the 15-year histories of selected diseases for participants, as previously described [20]. This was based on data from the Danish National Patient Registry, which records time, place, and primary/secondary diagnoses for all hospital visits in Denmark [21].

\section{Cytokine Autoantibodies}

$\mathrm{C}-\mathrm{aAb}$ levels were measured as previously described [22], using plasma from participants collected at date of DBDS inclusion. Plasma was collected in 2010, and c-aAb levels were measured in 2014. High levels of $\mathrm{c}-\mathrm{aAb}$ were defined as median fluorescence intensity (MFI) signals above the 99th percentile, to identify participants with c-aAb levels most likely to inhibit their target cytokines. Separate cutoff values were generated for men and women, due to differences in $\mathrm{c}-\mathrm{aAb}$ signals between the groups, as previously described [16]. Low $\mathrm{c}-\mathrm{aAb}$ levels were defined as MFI values below MFI +4 standard deviations (SD) of the negative control, and intermediary $\mathrm{c}-\mathrm{aAb}$ levels were defined as MFI values between the low and high MFI groups. 


\section{Antimicrobial Prescriptions}

Data on prescription of antimicrobials were obtained from the Danish National Prescription Registry (DNPR), which records all pharmacy prescriptions of drugs in Denmark, from 1994 and onwards [23]. Prescriptions of antibacterials in general $($ atc $=\mathrm{J} 01)$, tetracyclines $($ atc $=\mathrm{J} 01 \mathrm{~A})$, penicillin $($ atc $=\mathrm{J} 01 \mathrm{C})$, sulfonamides $($ atc $=\mathrm{J} 01 \mathrm{E})$, macrolides $($ atc $=\mathrm{J} 01 \mathrm{~F})$, antimycotics $($ atc $=\mathrm{J} 02$ ), and antivirals (atc $=\mathrm{J} 05$ ) were identified for the study participants. Follow-up began at the date of DBDS inclusion and ended at the first antimicrobial prescription being filled, censored at 1 year of follow-up. Follow-up was also censored in the event of death, emigration, or end of follow-up for prescription data (December 31, 2016). Depending on the type of prescription being analyzed, participant's prescription history was defined as having had any prescriptions of the investigated antimicrobials or specific prescriptions of these, e.g., penicillin, macrolides, sulfonamides, etc., within 1 year prior to c-aAb measurement/DBDS inclusion.

\section{Infection-Related Hospital Diagnoses}

The Danish National Patient Registry was also used to identify diagnoses related to infection for the study participants, using the same ICD-10 criteria as previously, with chronic infections being excluded [20]. Briefly, the diagnoses investigated covered primary and secondary diagnoses and underlying medical conditions and included ICD-10 codes concerning infections. Follow-up time was defined as time between DBDS inclusion/c-aAb measurement and the first infection-related diagnosis within a year, censored like prescription data.

\section{Self- Reported Health-Related Quality of Life Scores}

The questionnaire included the short form 12 (SF-12) instrument, a validated form of the SF-36 instrument for estimation of health-related quality of life (HRQL), summarized as mental and physical component scores (MCS and PCS) [24]. MCS combines scales for vitality, social functioning, role-emotional, and mental health, and PCS combines physical functioning, role-physical, bodily pain, and general health. Lower scores correspond to poorer self-reported health, with mean values of 52.8 MCS (standard deviation $(\mathrm{SD})=8.3$ ) and 51.0 PCS $(\mathrm{SD}=8.1)$ in Danish individuals [25].

\section{Statistical Analyses}

Due to previously described sex-specific variation regarding c-aAb distribution [16], descriptive data were stratified according to sex. Continuous normally distributed data were compared across sex by student's t-test (c-aAb MFI signals were log-transformed, to approximate normal distributions).
To investigate the possible cytokine-inhibitory effects of high levels of $\mathrm{c}-\mathrm{aAb}$ in healthy individuals, we applied a Cox proportional hazards model, with antimicrobial prescription and infectious diagnosis incidence as outcomes, as proxies for immune competence. Unadjusted and multivariate analyses adjusted for age, smoking, BMI, OC, and prescription history were conducted with high or intermediary levels of $\mathrm{c}-\mathrm{aAb}$ as independent variables. Covariates for the multivariable analysis were selected based on associations with either prescriptions or diagnoses, with a $p$ value of $\leq 0.05$ in univariate tests. The initial univariate tests included student's $\mathrm{t}$-tests to test for associations between $\mathrm{c}-\mathrm{aAb}$ and antimicrobial prescriptions/diagnoses within 1 year following inclusion (coded as 0/1 for no or any prescription/diagnosis) with continuous variables (age, BMI, etc.) and chi-squared tests to test for associations between $\mathrm{c}-\mathrm{aAb}$ and binary variables (smoking, sex, Charlson comorbidity score $>0$, etc.). The outcomes in Cox analyses, i.e., filing antimicrobial prescriptions or infectious diagnoses, were assessed within 1 year of $\mathrm{c}-\mathrm{aAb}$ measurement, using the low-level $\mathrm{c}-\mathrm{aAb}$ group as reference (coded as 0 for low c-aAb and 1 for intermediary or high c$\mathrm{aAb}$ levels, investigated separately). The investigated prescriptions were antibacterials in general (compound endpoint) and specifically penicillins, sulfonamides, macrolides, antivirals, tetracyclines, and antimycotics. These were the most common prescription categories within our dataset (data not shown).

$\mathrm{T}$ - and chi-squared tests were also used to investigate association between low, intermediary, and high c-aAb levels and the selected epidemiological covariates in the multivariate model. Participants with intermediary and high c-aAb levels were compared separately to participants with low c-aAb levels.

Association between $\mathrm{C}-\mathrm{aAb}$ levels was investigated using Spearman's rank correlation for nonparametric, continuous MFI signals, and chi-squared tests for associations between ordinal (coded as $2=$ high, $1=$ intermediary, and $0=$ low levels of $\mathrm{c}-\mathrm{aAb}$ ) and binary (coded as $1=$ high or $0=$ non-high levels of $\mathrm{c}-\mathrm{aAb}$ ) variables.

In further multivariate Cox proportional hazards models, we analyzed the influence of combined $\mathrm{c}-\mathrm{aAb}$ for infectious risk, by analyzing antimicrobial prescriptions stratified according to overlap between combinations of low, intermediary, and high levels of $\mathrm{c}-\mathrm{aAb}$. All possible $\mathrm{c}-\mathrm{aAb}$ combinations were tested, two at a time (e.g., IL- $1 \alpha$ and IL-6 c-aAb, IL- $1 \alpha$ and IL-10 c-aAb, etc.), and were adjusted as described above. Participants with low levels of both investigated $\mathrm{c}-\mathrm{aAb}$ were used as reference.

Logistic regressions were used to test for the predictive value of $\mathrm{c}-\mathrm{aAb}$ (independent variables) for MCS or PCS (dependent variables), applying the same covariates as for the prescription/diagnosis analyses. Dichotomized variables representing low and high MCS and PCS were generated, 
based on continuous MCS/PCS scores below the 10th or above the 90th percentiles, respectively. Separate high/low $\mathrm{MCS} / \mathrm{PCS}$ variables were generated for men and women and adjusted as described above in separate analyses.

All analyses were stratified by sex and performed using STATA (STATA/MP15 for PC, StataCorp, College Station, TX). $p$ values below 0.05 were considered statistically significant. Data used for the analyses of this manuscript are not publicly available as they utilized the health registers of Statistics Denmark, which are restricted to Danish researches with explicit permits.

\section{Ethics}

All participants provided oral and written informed consent at the time of inclusion. The study was approved by the Danish health research ethics committee system (RH30-4444 I-suite 00922), and the biobank and research database were approved by the Danish Data Protection Agency (P-2019-99).

\section{Results}

\section{Characteristics of the Cohort}

The sample population was 8972 participants from the Danish Blood Donor Study (DBDS) with measured levels of cytokine-specific autoantibodies (c-aAb) against IL- $1 \alpha$, IL6, IL-10, interferon $\alpha$ (IFN $\alpha$ ), and GM-CSF. A demographic overview of the participants with complete data for all variables $(n=8185)$ is shown in Table 1 . All displayed variables were associated with antibacterial prescription filings in univariate tests. The population comprised $48.0 \%$ women and $52.0 \%$ men. Men were significantly older than women and had a significantly higher mental component score (MCS), IL-1 $\alpha \mathrm{c}-\mathrm{aAb}$, and IL-10 c-aAb, whereas women had higher levels of IL-6 c-aAb ( $p=0.0016)$ and higher body mass index (BMI) and filled more antibacterial prescriptions within 1year follow-up (all $p<0.0001$ ). High levels of IL-1 $\alpha$, IL-6, and IL-10 c-aAb were positively associated with age, and high levels of IL-6 c-aAb were negatively associated with use of oral contraceptives (OC). These specific associations were not observed at the intermediary c-aAb level (Table S1). The degree of association between the five investigated $\mathrm{c}-\mathrm{aAb}$ seemed to vary depending on their levels. When expressed as continuous variables of measured MFI and as ordinal variables grouped into high, intermediary, or low levels, positive correlations between all c-aAb were observed (Table S2). For associations between high levels of $\mathrm{c}-\mathrm{aAb}$, specifically a significant association was only observed for GM-CSF and IL$1 \alpha$ specific c-aAb in men (Table S2a).

\section{C-aAb and Predicted Risk of Antimicrobial Prescriptions}

In the adjusted Cox proportional hazards models for women, high levels of IFN $\alpha \mathrm{c}-\mathrm{aAb}$ predicted increased risk of antibacterial prescriptions in general, as well as macrolide and sulfonamide prescriptions (Fig. 1A). GM-CSF c-aAb predicted increased risk of sulfonamide prescriptions (Table 2). In contrast, high levels of IL-10 c-aAb predicted reduced risk of antibacterial prescriptions in general and reduced risk of penicillin prescriptions (Table 2, Fig. 1B). These associations were observed with high levels of $\mathrm{c}-\mathrm{aAb}$, but not with intermediary levels.

In men, high $\mathrm{c}-\mathrm{aAb}$ levels did not predict prescription of any antimicrobials (Table 2).

In both men and women, high c-aAb levels neither predicted risk of antimycotic, tetracycline, nor antiviral prescriptions or infection-related diagnoses (data not shown).

\section{Cumulative c-aAb Incidence and Antimicrobial Prescriptions}

In women, a pattern emerged where high levels of IL-10 c$\mathrm{aAb}$ when combined with the presence of different levels of all the other $\mathrm{c}-\mathrm{aAb}$ remained associated with a lower predicted risk of antibacterial prescriptions, particularly when IL- $1 \alpha$ and GM-CSF c-aAb levels were low (Fig. 2). The low hazard ratio (HR) for antibacterial prescriptions induced by high levels of IL-10 c-aAb was further reduced when IL-10 c-aAb were combined with intermediary levels of IL-6 c-aAb (Fig. 2), whereas intermediary levels of IL- 6 c-aAb combined with low or intermediary levels of other $\mathrm{c}-\mathrm{aAb}$ (e.g., IFN $\alpha$ and GM-CSF $\mathrm{c}-\mathrm{aAb}$ ) were associated with $\mathrm{HR}<1$. Women with high levels of IFN $\alpha$ and GM-CSF c-aAb combined with intermediary levels of IL- $1 \alpha$, IFN $\alpha$, or GM-CSF displayed the highest HR for antibacterial prescriptions indicating an additive biologic effect of the $c-a A b$.

\section{Cytokine Autoantibodies and Self-Reported Mental and Physical Health}

In women, high levels of IL-10 c-aAb predicted a high (better) physical component score (PCS) (above the 90th percentile) (Fig. 3A, odds ratio $(\mathrm{OR})=3.87,95 \% \mathrm{CI}=1.54-9.72, p=$ 0.004).

In men, high levels of IL- $1 \alpha \mathrm{c}-\mathrm{aAb}$ predicted a lower (poorer) self-reported mental component score (MCS) (below the 10 th percentile) in the adjusted models $(\mathrm{OR}=3.02,95 \%$ $\mathrm{CI}=1.29-7.05, p=0.010)$.

IL-6, IFN $\alpha$, and GM-CSF, c-aAb were not associated with MCS or PCS for both men and women. 
Table 1 Characteristics of the study population

\begin{tabular}{|c|c|c|c|}
\hline & Women & Men & $p$ value ${ }^{\mathrm{a}}$ \\
\hline Number of participants & $3926(48.0 \%)$ & $4259(52.0 \%)$ & \\
\hline Age (years) ${ }^{b}$ & $38.5( \pm 12.3)$ & $40.7( \pm 12.2)$ & $<0.0001$ \\
\hline Smoking (current) & $700(17.8 \%)$ & $698(16.4 \%)$ & 0.084 \\
\hline BMI & $25.6( \pm 3.4)$ & $24.5( \pm 4.1)$ & $<0.0001$ \\
\hline Combined oral contraceptives (yes) ${ }^{\mathrm{c}}$ & $1086(27.7 \%)$ & - & \\
\hline $\mathrm{MCS}^{\mathrm{b}}$ & $52.1( \pm 6.7)$ & $53.3( \pm 6.2)$ & $<0.0001$ \\
\hline $\mathrm{PCS}^{\mathrm{b}}$ & $55.1( \pm 4.1)$ & $55.1( \pm 3.9)$ & 0.945 \\
\hline Filled at least one prescription for antibacterial ${ }^{\mathrm{d}}$ & $1425(36.3 \%)$ & $934(21.9 \%)$ & $<0.0001$ \\
\hline IL-1 $\alpha \mathrm{c}-\mathrm{aAb}$ signal (MFI) ${ }^{\mathrm{b}}$ & $520( \pm 1282)$ & $787( \pm 2037)$ & $<0.0001$ \\
\hline Cutoff for high levels (MFI) ${ }^{e}$ & 6909 & 11,765 & \\
\hline IL-6 c-aAb signal (MFI) ${ }^{b}$ & $666( \pm 1633)$ & $595( \pm 1656)$ & 0.0016 \\
\hline Cutoff for high levels (MFI) ${ }^{\mathrm{e}}$ & 8007 & 6993 & \\
\hline IL-10 c-aAb signal (MFI) ${ }^{b}$ & $129( \pm 387)$ & $143( \pm 403)$ & $<0.0001$ \\
\hline Cutoff for high levels (MFI) ${ }^{\mathrm{e}}$ & 881 & 1177 & \\
\hline IFN $\alpha \mathrm{c}-\mathrm{aAb}$ signal (MFI) ${ }^{\mathrm{b}}$ & $173( \pm 572)$ & $169( \pm 521)$ & 0.234 \\
\hline Cutoff for high levels (MFI) ${ }^{e}$ & 2397 & 1799 & \\
\hline GM-CSF c- signal MFI ${ }^{\mathrm{b}}$ & $288( \pm 1312)$ & $267(+1150)$ & 0.115 \\
\hline Cutoff for high levels (MFI) ${ }^{\mathrm{e}}$ & 5861 & 5287 & \\
\hline
\end{tabular}

${ }^{\text {a }}$ Student's t-test was used for comparison of continuous variables across sex (age, BMI, PCS, MCS, c-aAb levels), and chi-squared tests were used for comparison of binary variables (smoking, OC use, prescription history)

${ }^{\mathrm{b}}$ Data presented as mean $\pm \mathrm{SD}$

${ }^{\mathrm{c}}$ Data presented as percentage of population

${ }^{\mathrm{d}}$ Data presented as incidence proportion, within 1 year of follow-up

${ }^{\text {e }}$ 99th percentile of group MFI

\section{Discussion}

Cytokine autoantibodies are critical players in both immunodeficiencies and autoimmune disease, inflicting a variety of pro- or anti-inflammatory effects, depending on the context and their specificity. The present study investigated the association between different $\mathrm{c}-\mathrm{aAb}$ directed against pro- and antiinflammatory cytokines in healthy individuals and infectious
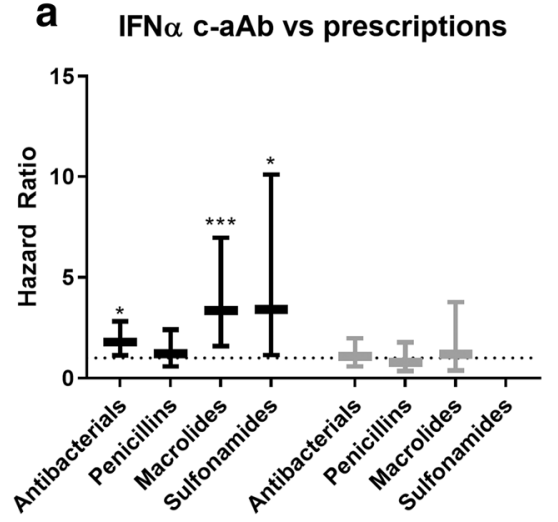

Fig. 1 IFN $\alpha$ and IL-10 c-aAb as predictors of antimicrobial prescriptions Multivariate Cox proportional hazards models were used to investigate high levels of (a) IFN $\alpha \mathrm{c}-\mathrm{aAb}$ and (b) IL-10 c-aAb as predictors of risk of filing prescriptions for antibacterials in general and, specifically, for penicillins, macrolides, and sulfonamides within 1 year of $\mathrm{c}-\mathrm{aAb}$ measurement. The models were adjusted for age, high levels of $\mathrm{c}-\mathrm{aAb}$, oral contraceptives, smoking, BMI, and 1 year prescription history of the

\section{b IL-10 C-aAb vs prescriptions}

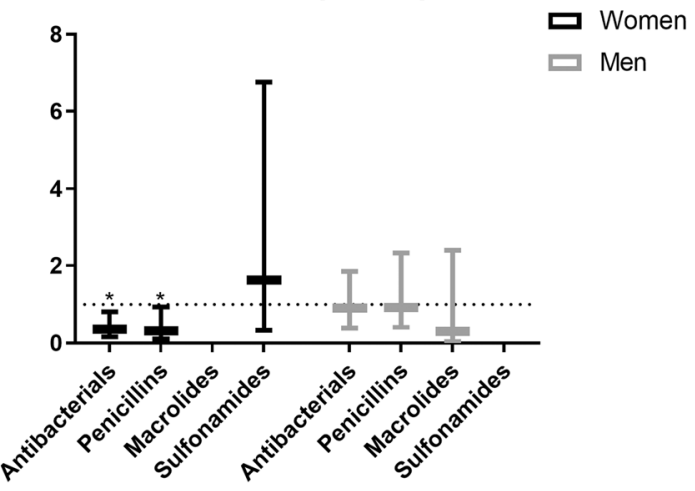

prescription subtype in question prior to $\mathrm{c}-\mathrm{aAb}$ measurement. For binary variables, high levels of $\mathrm{c}-\mathrm{aAb}$, usage of contraceptives, and having had at least one prescription a year before $\mathrm{c}-\mathrm{aAb}$ measurement were coded as 1 . High levels of $\mathrm{c}-\mathrm{aAb}$ were defined as MFI above the 99th percentile, calculated separately for women and men. Data are presented as HR with $95 \%$ Confidence Interval $(\mathrm{CI}) . *$ denotes a $p$ value of $\leq 0.05$, $* * *$ $p \leq 0.001$ 
Table $2 \mathrm{C}-\mathrm{aAb}$ as predictors of over-the-counter prescriptions within 1 year - women

\begin{tabular}{|c|c|c|c|c|c|c|}
\hline $\mathrm{C}-\mathrm{aAb}$ & Prescription & C-aAb level & $\mathrm{IR}^{\mathrm{a}}$ & Crude HR $(95 \% \mathrm{CI})$ & Adjusted HR $(95 \% \mathrm{CI})^{\mathrm{b}}$ & $p$ value \\
\hline \multirow[t]{8}{*}{$\mathrm{IFN} \alpha$} & \multirow[t]{2}{*}{ Antibacterials } & Intermediary $^{\mathrm{c}}$ & $1375 / 3002$ & $1.00(0.91-1.11)$ & $1.01(0.90-1.12)$ & 0.982 \\
\hline & & $\operatorname{High}^{\mathrm{d}}$ & $780 / 1683$ & $1.63(1.05-2.44)$ & $1.79(1.13-2.81)$ & 0.008 \\
\hline & \multirow[t]{2}{*}{ Penicillins } & Intermediary $^{c}$ & $1375 / 3335$ & $0.98(0.88-1.09)$ & $0.97(0.86-1.08)$ & 0.552 \\
\hline & & $\operatorname{High}^{\mathrm{d}}$ & $516 / 1862$ & $0.90(0.47-1.73)$ & $1.22(0.58-2.41)$ & 0.547 \\
\hline & \multirow[t]{2}{*}{ Macrolides } & Intermediary $^{c}$ & $267 / 3646$ & $0.97(0.77-1.21)$ & $0.95(0.74-1.22)$ & 0.682 \\
\hline & & $\operatorname{High}^{\mathrm{d}}$ & $157 / 2043$ & $3.60(1.96-6.77)$ & $3.61(1.70-7.67)$ & 0.001 \\
\hline & \multirow[t]{2}{*}{ Sulfonamides } & Intermediary $^{\mathrm{c}}$ & $139 / 3714$ & $1.16(0.84-1.60)$ & $1.27(0.90-1.79)$ & 0.171 \\
\hline & & $\operatorname{High}^{\mathrm{d}}$ & $73 / 2090$ & $2.84(1.05-7.94)$ & $3.58(1.19-10.64)$ & 0.026 \\
\hline \multirow[t]{8}{*}{ GM-CSF } & \multirow[t]{2}{*}{ Antibacterials } & Intermediary $^{c}$ & $1315 / 2890$ & $0.99(0.86-1.17)$ & $0.90(0.80-1.18)$ & 0.770 \\
\hline & & $\operatorname{High}^{\mathrm{d}}$ & $1277 / 2767$ & $1.44(0.91-2.26)$ & $1.57(0.96-2.64)$ & 0.070 \\
\hline & \multirow[t]{2}{*}{ Penicillins } & Intermediary $^{c}$ & $1315 / 3207$ & $0.99(0.84-1.17)$ & $0.97(0.81-1.18)$ & 0.811 \\
\hline & & $\operatorname{High}^{\mathrm{d}}$ & $757 / 2895$ & $1.24(0.68-2.23)$ & $1.32(0.70-2.69)$ & 0.353 \\
\hline & \multirow[t]{2}{*}{ Macrolides } & Intermediary $^{c}$ & $264 / 3500$ & $1.09(0.77-1.54)$ & $1.01(0.66-1.53)$ & 0.949 \\
\hline & & $\operatorname{High}^{\mathrm{d}}$ & $238 / 3162$ & $0.97(0.31-3.02)$ & $1.35(0.35-4.01)$ & 0.707 \\
\hline & \multirow[t]{2}{*}{ Sulfonamides } & Intermediary $^{c}$ & $135 / 3568$ & $0.63(0.34-1.18)$ & $0.55(0.26-1.16)$ & 0.117 \\
\hline & & $\operatorname{High}^{\mathrm{d}}$ & $131 / 3217$ & $2.74(0.98-7.19)$ & $3.21(1.12-9.18)$ & 0.029 \\
\hline \multirow[t]{8}{*}{ IL-10 } & \multirow[t]{2}{*}{ Antibacterials } & Intermediary $^{c}$ & $1364 / 2954$ & $1.02(0.93-1.13)$ & $0.99(0.89-1.11)$ & 0.839 \\
\hline & & $\operatorname{High}^{\mathrm{d}}$ & $827 / 1817$ & $0.38(0.18-0.81)$ & $0.36(0.16-0.81)$ & 0.015 \\
\hline & \multirow[t]{2}{*}{ Penicillins } & Intermediary $^{c}$ & $1364 / 3282$ & $1.01(0.91-1.12)$ & $0.98(0.88-1.09)$ & 0.697 \\
\hline & & $\operatorname{High}^{\mathrm{d}}$ & $522 / 1935$ & $0.37(0.14-0.97)$ & $0.22(0.05-0.89)$ & 0.032 \\
\hline & \multirow[t]{2}{*}{ Macrolides } & Intermediary $^{c}$ & $271 / 3591$ & $1.08(0.86-1.34)$ & $1.04(0.82-1.33)$ & 0.738 \\
\hline & & $\operatorname{High}^{\mathrm{d}}$ & - & - & - & \\
\hline & \multirow[t]{2}{*}{ Sulfonamides } & Intermediary $^{c}$ & $139 / 3663$ & $1.06(0.77-1.46)$ & $1.05(0.75-1.49)$ & 0.768 \\
\hline & & $\operatorname{High}^{\mathrm{d}}$ & $79 / 2161$ & $1.31(0.33-5.30)$ & $1.62(0.33-6.76)$ & 0.505 \\
\hline \multirow[t]{8}{*}{ IL-6 } & \multirow[t]{2}{*}{ Antibacterials } & Intermediary $^{c}$ & 1358 & $0.86(0.78-0.97)$ & $0.84(0.74-0.94)$ & 0.003 \\
\hline & & $\operatorname{High}^{\mathrm{d}}$ & $475 / 938$ & $0.79(0.52-1.35)$ & $0.73(0.41-1.31)$ & 0.301 \\
\hline & \multirow[t]{2}{*}{ Penicillins } & Intermediary $^{c}$ & $1358 / 3305$ & $0.86(0.78-0.97)$ & $0.85(0.76-0.95)$ & 0.214 \\
\hline & & $\operatorname{High}^{\mathrm{d}}$ & $304 / 1053$ & $0.62(0.33-1.31)$ & $0.49(0.24-1.38)$ & 0.119 \\
\hline & \multirow[t]{2}{*}{ Macrolides } & Intermediary $^{c}$ & $271 / 3609$ & $0.96(0.76-1.21)$ & $0.90(0.69-1.18)$ & 0.440 \\
\hline & & $\operatorname{High}^{\mathrm{d}}$ & $92 / 1166$ & $0.98(0.32-2.98)$ & $1.04(0.33-3.39)$ & 0.923 \\
\hline & \multirow[t]{2}{*}{ Sulfonamides } & Intermediary $^{c}$ & $141 / 3679$ & $0.82(0.59-1.14)$ & $0.89(0.63-1.28)$ & 0.557 \\
\hline & & $\operatorname{High}^{\mathrm{d}}$ & $50 / 1187$ & $0.56(0.09-4.10)$ & $0.80(0.11-5.89)$ & 0.824 \\
\hline \multirow[t]{8}{*}{$\mathrm{IL}-1 \alpha$} & \multirow[t]{2}{*}{ Antibacterials } & Intermediary $^{c}$ & $1378 / 2986$ & $0.98(0.89-1.08)$ & $1.01(0.90-1.12)$ & 0.921 \\
\hline & & $\operatorname{High}^{\mathrm{d}}$ & $733 / 1576$ & $0.75(0.42-1.32)$ & $0.94(0.49-1.68)$ & 0.844 \\
\hline & \multirow[t]{2}{*}{ Penicillins } & Intermediary $^{c}$ & $1295 / 3068$ & $0.97(0.88-1.07)$ & $0.99(0.88-1.10)$ & 0.813 \\
\hline & & $\operatorname{High}^{\mathrm{d}}$ & $479 / 1748$ & $0.69(0.33-1.45)$ & $0.87(0.41-1.85)$ & 0.717 \\
\hline & \multirow[t]{2}{*}{ Macrolides } & Intermediary $^{c}$ & $270 / 3635$ & $1.21(0.97-1.51)$ & $1.28(1.01-1.64)$ & 0.041 \\
\hline & & $\operatorname{High}^{\mathrm{d}}$ & $129 / 1927$ & $1.85(0.76-4.52)$ & $2.25(0.90-5.65)$ & 0.083 \\
\hline & \multirow[t]{2}{*}{ Sulfonamides } & Intermediary $^{c}$ & $136 / 3705$ & $0.93(0.68-1.28)$ & $0.88(0.62-1.26)$ & 0.497 \\
\hline & & $\operatorname{High}^{\mathrm{d}}$ & $74 / 1958$ & $0.64(0.09-4.56)$ & $0.64(0.09-4.47)$ & 0.618 \\
\hline
\end{tabular}

risk, assessed as time until the next prescription of antimicrobials. We found that high-titer levels of c-aAb against pro- and anti-inflammatory cytokines predicted increased and reduced risk, respectively, of prescriptions of various antimicrobials as a proxy for infectious risk. As we observed positive associations between the c-aAb MFI signals, we also studied the pairwise association of $\mathrm{c}-\mathrm{aAb}$ with prescriptions, which suggested a pattern of cumulative c-aAb effects. These findings suggest that $\mathrm{c}-\mathrm{aAb}$ in healthy individuals are present in levels that may have immunomodulatory effects, and in the following, we speculate on the possible mechanisms of these.

In line with prior studies [16], we found c-aAb levels to be associated with sex and age, as well as a novel association of IL-6 c-aAb and oral contraceptives. As such, we observed a 
Table $3 \mathrm{C}-\mathrm{aAb}$ as predictors of over-the-counter prescriptions within 1 year - men

\begin{tabular}{|c|c|c|c|c|c|c|}
\hline $\mathrm{C}-\mathrm{aAb}$ & Prescription & $\mathrm{C}-\mathrm{aAb}$ level & $\mathrm{C}-\mathrm{aAb}$ & Prescription & C-aAb level & $\begin{array}{l}\mathrm{C}- \\
\mathrm{aAb}\end{array}$ \\
\hline \multirow[t]{6}{*}{$\mathrm{IFN} \alpha$} & \multirow[t]{2}{*}{ Antibacterials } & Intermediary $^{c}$ & $905 / 3652$ & $0.91(0.81-1.03)$ & $0.87(0.76-1.00)$ & 0.050 \\
\hline & & $\operatorname{High}^{\mathrm{d}}$ & $527 / 2001$ & $1.12(0.62-1.95)$ & $1.08(0.58-1.98)$ & 0.810 \\
\hline & \multirow[t]{2}{*}{ Penicillins } & Intermediary $^{c}$ & $905 / 3810$ & $0.91(0.78-1.07)$ & $0.89(0.76-1.05)$ & 0.057 \\
\hline & & $\operatorname{High}^{\mathrm{d}}$ & $388 / 2373$ & $0.88(0.39-1.96)$ & $0.78(0.36-1.78)$ & 0.659 \\
\hline & \multirow[t]{2}{*}{ Macrolides } & Intermediary $^{c}$ & $258 / 3990$ & $0.78(0.62-1.01))$ & $0.80(0.62-1.04)$ & 0.096 \\
\hline & & $\operatorname{High}^{\mathrm{d}}$ & $158 / 2192$ & $0.97(0.31-3.04)$ & $1.17(0.36-3.76)$ & 0.941 \\
\hline \multirow[t]{6}{*}{ GM-CSF } & \multirow[t]{2}{*}{ Antibacterials } & Intermediary $^{c}$ & $874 / 3510$ & $1.01(0.84-1.21)$ & $0.89(0.71-1.10)$ & 0.286 \\
\hline & & $\operatorname{High}^{\mathrm{d}}$ & $768 / 3076$ & $0.71(0.34-1.49)$ & $0.78(0.30-1.66)$ & 0.530 \\
\hline & \multirow[t]{2}{*}{ Penicillins } & Intermediary $^{c}$ & $874 / 3663$ & $1.01(0.83-1.21)$ & $0.88(0.71-1.10)$ & 0.282 \\
\hline & & $\operatorname{High}^{\mathrm{d}}$ & $535 / 3209$ & $0.59(0.22-1.58)$ & $0.53(0.20-1.58)$ & 0.286 \\
\hline & \multirow[t]{2}{*}{ Macrolides } & Intermediary $^{\mathrm{c}}$ & $241 / 3842$ & $1.16(0.83-1.63)$ & $0.99(0.67-1.49)$ & 0.991 \\
\hline & & $\operatorname{High}^{\mathrm{d}}$ & $211 / 3374$ & $1.15(0.37-3.61)$ & $1.22(0.39-3.87)$ & 0.760 \\
\hline \multirow[t]{6}{*}{ IL-10 } & \multirow[t]{2}{*}{ Antibacterials } & Intermediary $^{\mathrm{c}}$ & $893 / 3602$ & $1.01(0.89-1.14)$ & $0.99(0.87-1.14)$ & 0.921 \\
\hline & & $\operatorname{High}^{\mathrm{d}}$ & $468 / 1871$ & $0.82(0.41-1.64)$ & $0.91(0.39-1.85)$ & 0.810 \\
\hline & \multirow[t]{2}{*}{ Penicillins } & Intermediary $^{\mathrm{c}}$ & $893 / 3757$ & $0.99(0.88-1.13)$ & $1.01(0.88-1.14)$ & 0.979 \\
\hline & & $\operatorname{High}^{\mathrm{d}}$ & $330 / 1950$ & $0.93(0.42-2.08)$ & $0.92(0.41-2.33)$ & 0.930 \\
\hline & \multirow[t]{2}{*}{ Macrolides } & Intermediary ${ }^{c}$ & $251 / 3937$ & $0.95(0.76-1.21)$ & $0.92(0.71-1.18)$ & 0.511 \\
\hline & & $\operatorname{High}^{\mathrm{d}}$ & $133 / 2047$ & $0.35(0.05-2.52)$ & $0.31(0.04-2.40)$ & 0.274 \\
\hline \multirow[t]{6}{*}{ IL-6 } & \multirow[t]{2}{*}{ Antibacterials } & Intermediary $^{c}$ & $897 / 3628$ & $0.99(0.87-1.14)$ & $1.02(0.88-1.18)$ & 0.770 \\
\hline & & $\operatorname{High}^{\mathrm{d}}$ & $302 / 1214$ & $1.49(0.87-2.51)$ & $1.34(0.75-2.42)$ & 0.316 \\
\hline & \multirow[t]{2}{*}{ Penicillins } & Intermediary $^{c}$ & $897 / 3788$ & $0.98(0.86-1.12)$ & $1.01(0.88-1.17)$ & 0.842 \\
\hline & & $\operatorname{High}^{\mathrm{d}}$ & $219 / 1261$ & $1.19(0.60-2.40)$ & $1.14(0.57-2.31)$ & 0.822 \\
\hline & \multirow[t]{2}{*}{ Macrolides } & Intermediary $^{\mathrm{c}}$ & $254 / 3963$ & $0.97(0.76-1.25)$ & $0.94(0.72-1.23)$ & 0.656 \\
\hline & & $\operatorname{High}^{\mathrm{d}}$ & $87 / 1325$ & $1.03(0.33-3.24)$ & $1.44(0.45-4.65)$ & 0.534 \\
\hline \multirow[t]{6}{*}{ IL-1 $\alpha$} & \multirow[t]{2}{*}{ Antibacterials } & Intermediary $^{c}$ & $905 / 3647$ & $1.04(0.92-1.19)$ & $1.05(0.91-1.21)$ & 0.488 \\
\hline & & $\operatorname{High}^{\mathrm{d}}$ & $417 / 1737$ & $0.57(0.26-1.27)$ & $0.48(0.21-1.07)$ & 0.086 \\
\hline & \multirow[t]{2}{*}{ Penicillins } & Intermediary $^{\mathrm{c}}$ & $905 / 3805$ & $1.04(0.92-1.19)$ & $1.06(0.92-1.22)$ & 0.385 \\
\hline & & $\operatorname{High}^{\mathrm{d}}$ & $288 / 1809$ & $0.72(0.30-1.73)$ & $0.61(0.23-1.57)$ & 0.329 \\
\hline & \multirow[t]{2}{*}{ Macrolides } & Intermediary $^{\mathrm{c}}$ & $256 / 3985$ & $0.84(0.67-1.07)$ & $0.85(0.65-1.10)$ & 0.215 \\
\hline & & $\operatorname{High}^{\mathrm{d}}$ & $131 / 1887$ & $0.98(0.34-3.09)$ & $0.86(0.32-2.85)$ & 0.814 \\
\hline
\end{tabular}

${ }^{\mathrm{a}} I R$ incidence rate per observed person-years, for adjusted analyses, $N$ number of cases for adjusted analyses ${ }^{\mathrm{b}}$

${ }^{\mathrm{b}} \mathrm{Cox}$ regressions were performed using intermediary/high levels of c-aAb vs low levels as predictors vs low c$\mathrm{aAb}$ levels and adjusted for age, smoking, BMI, combined oral contraceptives, and 1-year prescription history prior to $\mathrm{c}-\mathrm{aAb}$ measurement/DBDS inclusion

${ }^{\mathrm{c}}$ Intermediary c-aAb levels (negative control $+4 \mathrm{SD}<\mathrm{MFI}<99$ th percentile)

${ }^{\mathrm{d}}$ High c-aAb levels (MFI > 99th percentile) strong sex interaction for $\mathrm{c}-\mathrm{aAb}$ and our investigated outcomes. In women, high levels of IFN $\alpha \mathrm{c}-\mathrm{aAb}$ were associated with increased risk of macrolide, sulfonamide, and antibacterial prescriptions in general. Previous studies have associated IFN $\alpha \mathrm{c}-\mathrm{aAb}$ levels with the occurrence of viral infections and have established a strong genetic component for the occurrence of this $\mathrm{c}-\mathrm{aAb}$ [26-29]. This makes it a prime candidate for further studies as a risk factor for infection. The finding in the present study that INF $\alpha \mathrm{c}-\mathrm{aAb}$ levels associate with various antimicrobial prescriptions (indicative of different infections, i.e., respiratory infections and urinary tract infections) indicates a broader biologic effect of IFN $\alpha \mathrm{c}-\mathrm{aAb}$ though causality remains to be established. GM-CSF c-aAb have also been associated with multiple variants of opportunistic infections [30], and here we observed high levels of $\mathrm{c}-\mathrm{aAb}$ to be associated with an increased risk of sulfonamide prescriptions in women. Interestingly, despite the previously observed association between IL-6 c-aAb and low levels of CRP [16], high levels of IL-6 c-aAb did not predict higher or lower risk of filing prescriptions in this study, which may reflect the pleiotropic roles of IL-6 as both a pro- and antiinflammatory cytokine [31]. Furthermore, c-aAb have in some reports been observed to act as agonists of their target cytokine function [32], adding further complexity to our findings. 
C-aAb overlap vs antibacterial prescriptions, Women

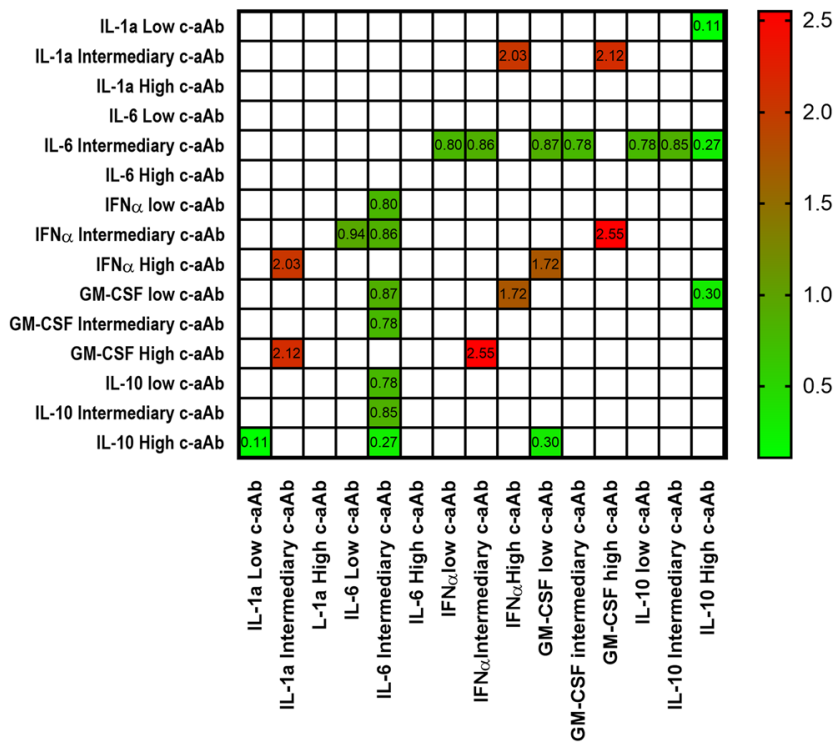

Fig. $2 \mathrm{C}-\mathrm{aAb}$ overlap vs antimicrobial prescriptions in women Multivariate Cox proportional hazards models were used to investigate the intersection of low/intermediary and high levels of IL- $1 \alpha$, GM-CSF, IL-6, IFN $\alpha$, and IL-10 c-aAb as predictors of risk of filing antimicrobial prescriptions within 1 year of $\mathrm{c}-\mathrm{aAb}$ measurement. Analyses were performed for the interaction of two $\mathrm{c}-\mathrm{aAb}$ at a time, with HR calculated using participants with low levels of both investigated c-aAb as a baseline. The models were adjusted for age, high levels of $\mathrm{c}-\mathrm{aAb}$, oral contraceptives, smoking, BMI, and 1 year prescription history of the prescription subtype in question prior to $\mathrm{c}-\mathrm{aAb}$ measurement. For binary variables, high levels of $\mathrm{c}-\mathrm{aAb}$, usage of contraceptives, and having had at least one prescription a year before $\mathrm{c}-\mathrm{aAb}$ measurement were coded as 1 . High levels of $\mathrm{c}-\mathrm{aAb}$ were defined as MFI above the 99th percentile, intermediary c-aAb levels were defined as MFI values $>$ negative control $+4 \mathrm{SD}$ and $<$ the 99th percentile, and low levels were defined as MFI values $\leq$ negative control $+4 \mathrm{SD}$. Data are presented as HR. All displayed HR corresponds to $p$ values of $\leq 0.05$

Notably, high levels of IL-10 c-aAb were associated with lower risk of penicillin and general antibacterial prescriptions in women, also when combined with other c-aAb. It may be speculated that the supposedly protective effects of IL-10 caAb may be due to suppression of the anti-inflammatory regulator IL-10, hereby creating a more pro-inflammatory and more potent antimicrobial environment, as previously reported [3].

As an alternative outcome to approximate c-aAb influence on participant health, we investigated the association to selfreported PCS and MCS health scores, hypothesizing that participants with c-aAb-mediated immunomodulation may present with lower or higher scores. Notably, we observed the same pattern for self-reported health scores as we did for antibacterial prescriptions, so women with a high level of IL-10 c-aAb had increased odds of high (good) PCS scores, again suggesting a potentially protective and positive effect of IL-10 c-aAb. It should be emphasized that the effects of IL-10 c-aAb are likely highly context-specific, as another previous study from our research group reported that IL-10 c-aAb were associated with increased risk of major adverse cardiovascular events (MACE) in kidney-transplanted patients, an outcome that is intrinsically linked to a pro-inflammatory environment [33]. Thus, though protective in regard to infections in healthy individuals, IL-10 c$\mathrm{aAb}$ may constitute a risk factor for patients in whom excess inflammation may tip a balance towards adverse events such as organ transplant patients and autoimmune disease in general, again due to the cytokines' many immunoregulatory functions $[34,35]$. Conversely, c-aAb against pro-inflammatory cytokines IFN $\alpha$ and IL- $1 \alpha$ have been linked to improved prognoses in autoimmune disease [6].

The observed associations between IL-10 c-aAb, prescriptions, and self-reported health being specific to women are notable, considering that women in our study had lower levels of detectable IL-10 c-aAb compared to men [16]. This finding may suggest a higher tolerance for IL-10 $\mathrm{c}-\mathrm{aAb}$ in men. Conversely, we observed an association between high levels of IL- $1 \alpha \mathrm{c}-\mathrm{aAb}$ and a low (poor) MCS in men, indicating increased sensitivity for this $\mathrm{c}-\mathrm{aAb}$ in men. It should be noted that the significant effects were only observed for the highest levels of $\mathrm{c}-\mathrm{aAb}$, suggesting that these titers are a requirement for possible functional cytokine suppression and ensuing biologic effects.

The limited association between several high-titer $\mathrm{c}-\mathrm{aAb}$ and infection or health-related outcomes may emphasize the redundancy of the human cytokine network, as c-aAbmediated functional inhibition of just one cytokine may not be enough to challenge immune competence. Though there was a positive association between $\mathrm{c}-\mathrm{aAb}$, this seemed limited to their intermediary levels at most, with a near-total lack of association between high-titer $\mathrm{c}-\mathrm{aAb}$ in individuals. This combined with their rarity means that we were unable to investigate cumulative incidences of high c-aAb levels as predictors of infection risk in this study. However, we assessed c-aAb overlap between the low/intermediary/high levels, and in women, we observed distinct patterns of higher or lower predicted risk of antibacterial prescriptions. The presence of intermediary or high levels of c-aAb against anti-inflammatory cytokine IL-10 and pleiotropic IL-6 coincided with reduced

Fig. $3 \mathrm{C}-\mathrm{aAb}$ as predictors of high/low self-reported physical/mental health

Multivariate logistic regression models were used to investigate high levels $\mathrm{c}-\mathrm{aAb}$ as predictors of having high or low scores of PCS and MCS. Independent variables include age, high levels of $\mathrm{c}-\mathrm{aAb}$, smoking, BMI, oral contraceptives, and 1 year prescription history of antibacterials. For binary variables, having high levels of c-aAb, usage of contraceptives, and having had at least one prescription a year before c$\mathrm{aAb}$ measurement were coded as 1. High and low levels of PCS and MCS were defined as scores above or below the 90th and 10th percentiles, respectively. Intermediary c-aAb levels were defined as MFI values $>$ negative control $+4 \mathrm{SD}$ and $<$ the 99th percentile. High levels of $\mathrm{c}-\mathrm{aAb}$ were defined as MFI above the 99th percentile with all high/low binary variables calculated separately for women and men. Data are presented as OR with $95 \%$ Confidence Interval (CI). * denotes a $p$ value of $\leq 0.05$ 
Health-related quality of life. Physical and mental component score

Women

Men

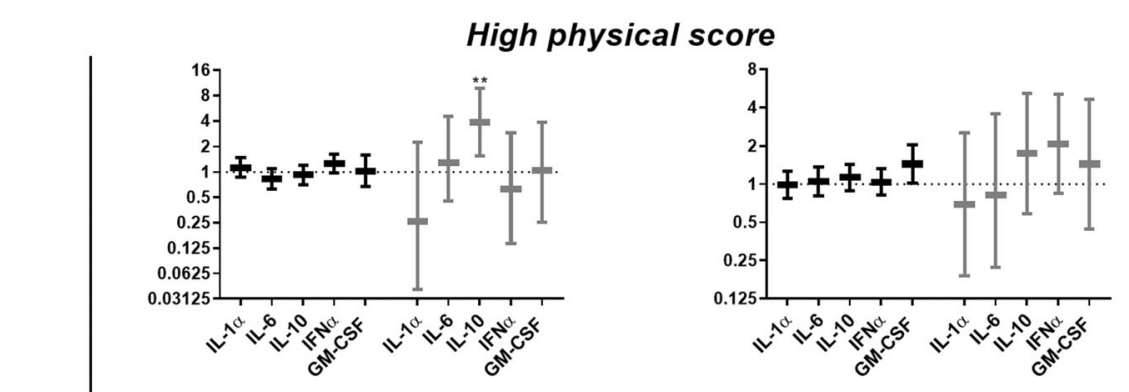

\section{Low physical_score}
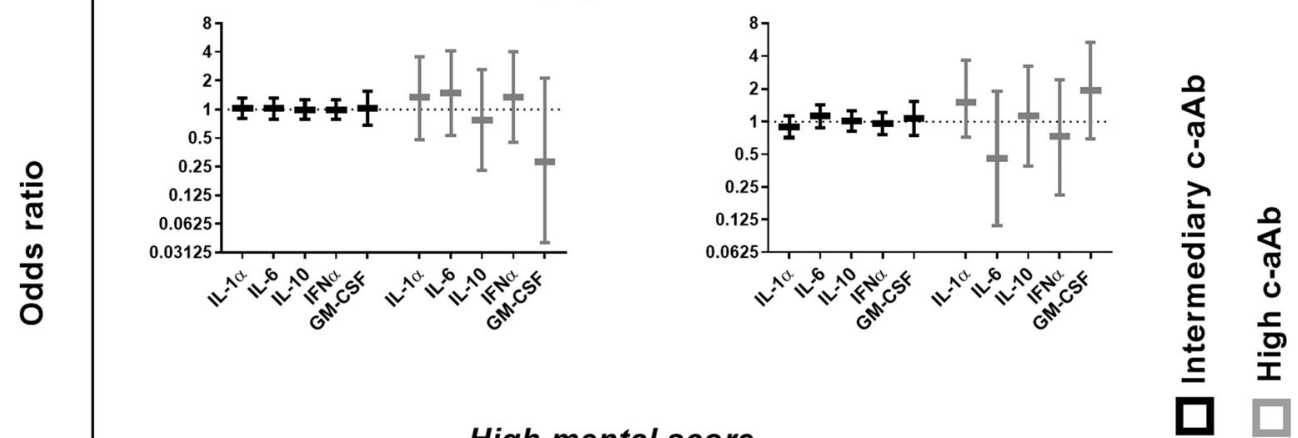

\section{High mental score}
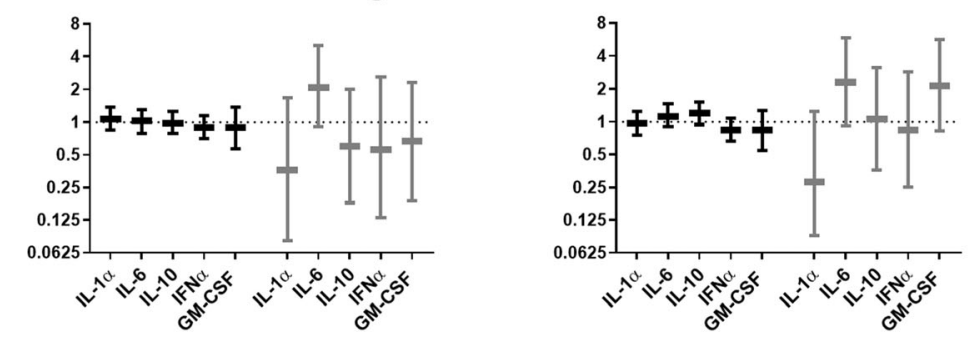

\section{Low mental score}
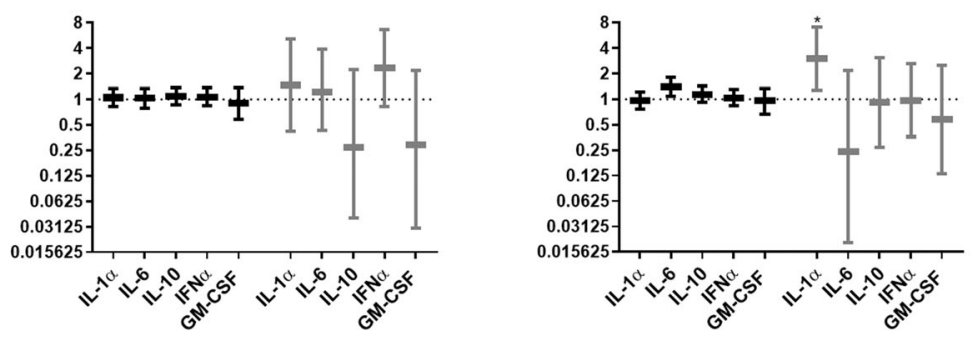

C-aAb specificity

risk of antibacterial prescriptions, which may again be due to promoting an increasingly pro-inflammatory environment. Conversely, participants with high/intermediary levels of pro-inflammatory specific $\mathrm{c}-\mathrm{aAb}$ had increased risk of antimicrobial prescriptions, suggesting additive immunosuppression by presence of several $\mathrm{c}-\mathrm{aAb}$ against proinflammatory cytokines. Together, these data suggest that there may be cumulative immunomodulatory effects of 
$\mathrm{c}-\mathrm{aAb}$, depending on the specificity of the high-titer $\mathrm{c}-\mathrm{aAb}$ present.

Overall, the data from the present study suggest that high levels of $\mathrm{c}-\mathrm{aAb}$ mediate target-specific immunomodulation in healthy individuals. This, along with the many studies demonstrating associations between $\mathrm{c}-\mathrm{aAb}$ and opportunistic infections $[6,8,28,30]$, raises further concern for the influence of high levels of $\mathrm{c}-\mathrm{aAb}$ in immunocompromised individuals. Future studies in such patients are warranted to determine the impact of high levels of $\mathrm{c}-\mathrm{aAb}$ on the immune system and overall patient health, as the influence of $\mathrm{c}-\mathrm{aAb}$ on patient outcome which may be very different in this context. Given that immunological profiling, including cytokine assessment in plasma and stimulated cell cultures, is increasingly used as a precision medicine approach to improve care of immunocompromised patients, the present study supports the notion that monitoring $\mathrm{c}-\mathrm{aAb}$ may be a critical component of such a profile.

In terms of strengths and limitations, this is to our knowledge the first study that investigates the association between cytokine autoantibody levels and infection risk and general health in healthy individuals. Because the study is crosssectional and population-based and because outcomes are collected systematically and through public registries, we believe there are no systematic biases that could have interfered with the internal validity of the study. Though the use of a healthy and highly characterized blood donor cohort minimizes potential confounding effects of chronic illness or comorbidity, the generalizability of the results might be reduced because blood donors are generally healthier and have a healthier lifestyle as compared with the general population [36], which may result in higher than average self-perceived health scores compared to the general population [37]. Furthermore, it is critical to emphasize that the results of the study are purely based on associations and not causal relationships, which should be taken into account when interpreting the results. The number of analyses performed may also create a risk for Type I errors through multiple testing. Finally, there is also the possibility of systemic biases in the case of a self-reported outcome such as BMI, where weight may be underreported.

\section{Conclusions}

In conclusion, we demonstrated that high levels of c-aAb specific for several pro-inflammatory cytokines predicted increased risk of infection in healthy individuals, whereas high levels of c-aAb against anti-inflammatory IL-10 predicted lower infection risk, as well as increased self-reported physical health.

These findings indicate that $\mathrm{c}-\mathrm{aAb}$ in healthy individuals are present in levels that may have immunomodulatory effects, depending on the specific c-aAb-targeted cytokine.
Overall, the findings emphasize that high-titer cytokine autoantibodies are biologically active in healthy individuals.

Acknowledgments We would like to thank the contribution of the Danish blood donors for making this study possible.

Authors' Contributions The following authors have contributed to the writing of this manuscript:

Acquisition of funding: Jakob H. von Stemann, Ole B. Pedersen, Henrik Hjalgrim, Christian Erikstrup, and Henrik Ullum.

Study design and planning: Jakob H. von Stemann, Morten B. Hansen, and Sisse R. Ostrowski.

Data gathering: Jakob H. von Stemann, Ole B. Pedersen, Henrik Hjalgrim, Christian Erikstrup, Henrik Ullum, Lise W. Thørner, Margit AH. Larsen, Kristoffer S. Burgdorf, and Erik Sørensen.

Data analysis: Jakob H. von Stemann, Ole B. Pedersen, Henrik Hjalgrim, Christian Erikstrup, Henrik Ullum, Morten B. Hansen, and Sisse R. Ostrowski.

Writing of manuscript: Jakob H. von Stemann, Morten B. Hansen, and Sisse R. Ostrowski.

Funding Information The present study has been funded by the Copenhagen University Hospital Research Fund, and DBDS has been funded by the Danish Regions and Bio- and Genome Bank Denmark.

\section{Compliance with Ethical Standards}

Conflict of Interest The authors declare that they have no conflict of interest.

Open Access This article is licensed under a Creative Commons Attribution 4.0 International License, which permits use, sharing, adaptation, distribution and reproduction in any medium or format, as long as you give appropriate credit to the original author(s) and the source, provide a link to the Creative Commons licence, and indicate if changes were made. The images or other third party material in this article are included in the article's Creative Commons licence, unless indicated otherwise in a credit line to the material. If material is not included in the article's Creative Commons licence and your intended use is not permitted by statutory regulation or exceeds the permitted use, you will need to obtain permission directly from the copyright holder. To view a copy of this licence, visit http://creativecommons.org/licenses/by/4.0/.

\section{References}

1. Altan-Bonnet G, Mukherjee R. Cytokine-mediated communication: a quantitative appraisal of immune complexity. Nat Rev Immunol. 2019;19(4):205-17.

2. Galle P, Hougs L, Barington T, Svenson M, Svejgaard A, Thomsen AR, et al. Knocking out IL-6 by vaccination. Eur J Immunol. 2004;34(1):291-300.

3. de Lemos RC, Galle P, Pedersen BK, Hansen MB. A state of acquired IL-10 deficiency in $0.4 \%$ of Danish blood donors. Cytokine. 2010;51(3):286-93.

4. Svenson M, Poulsen LK, Fomsgaard A, Bendtzen K. IgG autoantibodies against interleukin 1 alpha in sera of normal individuals. Scand J Immunol. 1989;29(4):489-92.

5. Svenson M, Hansen MB, Ross C, Diamant M, Rieneck K, Nielsen $\mathrm{H}$, et al. Antibody to granulocyte-macrophage colony-stimulating 
factor is a dominant anti-cytokine activity in human IgG preparations. Blood. 1998;91(6):2054-61.

6. Vincent T, Plawecki M, Goulabchand R, Guilpain P, Eliaou JF. Emerging clinical phenotypes associated with anti-cytokine autoantibodies. Autoimmun Rev. 2015;14(6):528-35.

7. Nanki T, Onoue I, Nagasaka K, Takayasu A, Ebisawa M, Hosoya T, et al. Suppression of elevations in serum $\mathrm{C}$ reactive protein levels by anti-IL-6 autoantibodies in two patients with severe bacterial infections. Ann Rheum Dis. 2013;72(6):1100-2.

8. Browne SK, Holland SM. Anti-cytokine autoantibodies explain some chronic mucocutaneous candidiasis. Immunol Cell Biol. 2010;88(6):614-5.

9. Kitamura T, Tanaka N, Watanabe J, Uchida, Kanegasaki S, Yamada $\mathrm{Y}$, et al. Idiopathic pulmonary alveolar proteinosis as an autoimmune disease with neutralizing antibody against granulocyte/ macrophage colony-stimulating factor. J Exp Med. 1999;190(6): 875-80.

10. Ben-Dov I, Segel MJ. Autoimmune pulmonary alveolar proteinosis: clinical course and diagnostic criteria. Autoimmun Rev. 2014;13(4-5):513-7.

11. Bousfiha A, Jeddane L, Picard C, Ailal F, Bobby Gaspar H, Al-Herz W, et al. The 2017 IUIS phenotypic classification for primary immunodeficiencies. J Clin Immunol. 2018;38(1):129-43.

12. Graudal NA, Svenson M, Tarp U, Garred P, Jurik AG, Bendtzen K. Autoantibodies against interleukin 1alpha in rheumatoid arthritis: association with long term radiographic outcome. Ann Rheum Dis. 2002;61(7):598-602.

13. Burbelo PD, Browne S, Holland SM, Iadarola MJ, Alevizos I. Clinical features of Sjogren's syndrome patients with autoantibodies against interferons. Clin Transl Med. 2019;8(1):1.

14. Notarangelo LD, Holland SM. Friendly fire: anti-cytokine antibodies elicited by microbes. Nat Med. 2016;22(9):973-5.

15. Kisand K, Link M, Wolff AS, Meager A, Tserel L, Org T, et al. Interferon autoantibodies associated with AIRE deficiency decrease the expression of IFN-stimulated genes. Blood. 2008;112(7):265766.

16. von Stemann JH, Rigas AS, Thorner LW, Rasmussen DGK, Pedersen OB, Rostgaard K, et al. Prevalence and correlation of cytokine-specific autoantibodies with epidemiological factors and C-reactive protein in 8,972 healthy individuals: results from the Danish blood donor study. PLoS One. 2017;12(6):e0179981.

17. Pedersen OB, Erikstrup C, Kotze SR, Sorensen E, Petersen MS, Grau K, et al. The Danish blood donor study: a large, prospective cohort and biobank for medical research. Vox Sang. 2012;102(3): 271.

18. Hansen TF, Banasik K, Erikstrup C, Pedersen OB, Westergaard D, Chmura PJ, et al. DBDS genomic cohort, a prospective and comprehensive resource for integrative and temporal analysis of genetic, environmental and lifestyle factors affecting health of blood donors. BMJ Open. 2019;9(6):e028401.

19. Charlson ME, Pompei P, Ales KL, MacKenzie CR. A new method of classifying prognostic comorbidity in longitudinal studies: development and validation. J Chronic Dis. 1987;40(5):373-83.

20. Kaspersen KA, Pedersen OB, Petersen MS, Hjalgrim H, Rostgaard $\mathrm{K}$, Moller BK, et al. Obesity and risk of infection: results from the Danish blood donor study. Epidemiology. 2015;26(4):580-9.

21. Lynge E, Sandegaard JL, Rebolj M. The Danish National Patient Register. Scand J Public Health. 2011;39(7 Suppl):30-3.

22. Guldager DK, von Stemann JH, Larsen R, Bay JT, Galle PS, Svenson M, et al. A rapid, accurate and robust particle-based assay for the simultaneous screening of plasma samples for the presence of five different anti-cytokine autoantibodies. J Immunol Methods. 2015;425:62-8.

23. Kildemoes HW, Sorensen HT, Hallas J. The Danish National Prescription Registry. Scand J Public Health. 2011;39(7 Suppl): 38-41.

24. Ware J Jr, Kosinski M, Keller SD. A 12-item short-form health survey: construction of scales and preliminary tests of reliability and validity. Med Care. 1996;34(3):220-33.

25. Gandek B, Ware JE, Aaronson NK, Apolone G, Bjorner JB, Brazier $\mathrm{JE}$, et al. Cross-validation of item selection and scoring for the SF12 health survey in nine countries: results from the IQOLA project. International quality of life assessment. J Clin Epidemiol. 1998;51(11):1171-8.

26. Meager A, Visvalingam K, Peterson P, Moll K, Murumagi A, Krohn K, et al. Anti-interferon autoantibodies in autoimmune polyendocrinopathy syndrome type 1. PLoS Med. 2006;3(7):e289.

27. Strobel P, Murumagi A, Klein R, Luster M, Lahti M, Krohn K, et al. Deficiency of the autoimmune regulator AIRE in thymomas is insufficient to elicit autoimmune polyendocrinopathy syndrome type 1 (APS-1). J Pathol. 2007;211(5):563-71.

28. Burbelo PD, Browne SK, Sampaio EP, Giaccone G, Zaman R, Kristosturyan E, et al. Anti-cytokine autoantibodies are associated with opportunistic infection in patients with thymic neoplasia. Blood. 2010;116(23):4848-58.

29. Walter JE, Rosen LB, Csomos K, Rosenberg JM, Mathew D, Keszei M, et al. Broad-spectrum antibodies against self-antigens and cytokines in RAG deficiency. J Clin Invest. 2015;125(11): 4135-48.

30. Browne SK. Anticytokine autoantibody-associated immunodeficiency. Annu Rev Immunol. 2014;32:635-57.

31. Scheller J, Chalaris A, Schmidt-Arras D, Rose-John S. The pro- and anti-inflammatory properties of the cytokine interleukin-6. Biochim Biophys Acta. 2011;1813(5):878-88.

32. Spohn G, Arenas-Ramirez N, Bouchaud G, Boyman O. Endogenous polyclonal anti-IL-1 antibody responses potentiate IL-1 activity during pathogenic inflammation. J Allergy Clin Immunol. 2017;139(6):1957-65 e3.

33. Lund KP, von Stemann JH, Eriksson F, Hansen MB, Pedersen BK, Sorensen SS, et al. IL-10-specific autoantibodies predict major adverse cardiovascular events in kidney transplanted patients - a retrospective cohort study. Transpl Int. 2019.

34. Baglaenko Y, Manion KP, Chang NH, Gracey E, Loh C, Wither JE. IL-10 production is critical for sustaining the expansion of CD5+ B and NKT cells and restraining autoantibody production in congenic lupus-prone mice. PLoS One. 2016;11(3):e0150515.

35. O'Garra A, Vieira P. T(H)1 cells control themselves by producing interleukin-10. Nat Rev Immunol. 2007;7(6):425-8.

36. Atsma F, de Vegt $\mathrm{F}$. The healthy donor effect: a matter of selection bias and confounding. Transfusion. 2011;51(9):1883-5.

37. Didriksen M, Allen RP, Burchell BJ, Thorner LW, Rigas AS, Di Angelantonio E, et al. Restless legs syndrome is associated with major comorbidities in a population of Danish blood donors. Sleep Med. 2018;45:124-31.

Publisher's Note Springer Nature remains neutral with regard to jurisdictional claims in published maps and institutional affiliations. 